\title{
Anomalous polarization conversion in arrays of ultrathin ferromagnetic nanowires
}

\author{
Andrey A. Stashkevich, ${ }^{1,2}$ Yves Roussigné, ${ }^{1}$ Alexander N. Poddubny,,${ }^{3,4,}{ }^{*}$ S.-M. Chérif, ${ }^{1}$ Y. Zheng, ${ }^{5}$ Franck Vidal, ${ }^{5}$ \\ Ilya V. Yagupov, ${ }^{3}$ Alexei P. Slobozhanyuk, ${ }^{3}$ Pavel A. Belov, ${ }^{3}$ and Yuri S. Kivshar ${ }^{6,3}$ \\ ${ }^{1}$ LSPM (CNRS-UPR 3407), Université Paris 13, Sorbonne Paris Cité, 93430 Villetaneuse, France \\ ${ }^{2}$ International Laboratory MultiferrLab, ITMO University, St. Petersburg 197101, Russia \\ ${ }^{3}$ ITMO University, St. Petersburg 197101, Russia \\ ${ }^{4}$ Ioffe Institute, St. Petersburg 194021, Russia \\ ${ }_{5}^{5}$ Institut des NanoSciences de Paris, UMR CNRS 7588, UPMC Université Paris 6, 4 Place Jussieu, 75005 Paris, France \\ ${ }^{6}$ Nonlinear Physics Center and ARC Center of Excellence CUDOS, Australian National University, Canberra ACT 0200, Australia
}

(Received 10 June 2015; revised manuscript received 8 November 2015; published 28 December 2015)

\begin{abstract}
We study the optical properties of arrays of ultrathin cobalt nanowires by means of the Brillouin scattering of light on magnons. We employ the Stokes/anti-Stokes scattering asymmetry to probe the circular polarization of a local electric field induced inside nanowires by linearly polarized light waves. We observe the anomalous polarization conversion of the opposite sign than that in a bulk medium or thick nanowires with a great enhancement of the degree of circular polarization attributed to the unconventional refraction in a nanowire medium. A rigorous simulation of the electric field polarization as a function of the wire diameter and spacing reveals the reversed polarization for a thin sparse wire array, in full quantitative agreement with experimental results.
\end{abstract}

DOI: 10.1103/PhysRevB.92.214436

PACS number(s): 78.67.Uh, 42.79.Dj, 78.20.Ls, 78.35.+c

\section{INTRODUCTION}

The study of the magneto-optical response of tailored nanostructures is a focus of active research of nanostructured materials [1-3]. Nonmagnetic metallic nanowires are well known in optics, and they are employed as building blocks of the so-called wire metamaterials [4]. Such structures demonstrate many unusual properties, including negative refraction [5], enhanced sensing [6], super lensing [7], strong nonlocal effects [8], and nonlinearities [9], and they can boost light-matter interaction in the regime of the hyperbolic dispersion [10].

Here, we study magneto-optical (MO) properties of ultrathin ferromagnetic Co nanowire arrays (see Fig. 1) by means of polarization-resolved Brillouin light scattering (BLS) on magnons [11-13]. In sharp contrast to the previous studies of ferromagnetic rings [14] and nanowires with diameter $D \gtrsim 20 \mathrm{~nm}[15,16]$, here we investigate thin nanowires with $D \sim 4.8 \mathrm{~nm}$ spaced on a distance $\sim 17 \mathrm{~nm}$ (see insets in Fig. 1). Since the studied wires are thinner than the skin depth $(\gtrsim 10 \mathrm{~nm})$ and have very high aspect ratio of $\sim 100$, one can expect magneto-optical properties quite different from those reported in the literature.

The vital importance of the sample geometry was prompted by the somewhat inconclusive results reported in our earlier paper [16]. On the one hand, in an array of "large and stumpy rods" $(D=70 \mathrm{~nm}, L=175 \mathrm{~nm}$, aspect ratio $L / D=2.5)$, one of the submaxima in a complex BLS line structure did demonstrate a behavior that was unusual compared to bulk magnetic films [11,17]. On the other hand, the alternative structure of a more classical nanowire configuration $(D=$ $20 \mathrm{~nm}, L=175 \mathrm{~nm}$, aspect ratio $L / D=9$ ) manifested a perfectly conventional BLS signature.

The three specific unconventional features of our study, discussed below in detail, are (i) use of the tailor-made sample with very thin nanowires and large aspect ratio of $\sim 100$, (ii) use

\footnotetext{
*a.poddubny@phoi.ifmo.ru
}

of the BLS scattering to probe photons rather than magnons, since the magnon properties are well defined for thin wires, and (iii) strong and reversed circular polarization of photons found inside the nanowires. Namely, we reveal a striking anomaly of the Stokes/anti-Stokes pattern in the spectra of BLS from magnons in the structures with thin and long wires. First, its asymmetry is inverted, and, second, it is considerably larger than in continuous Co film. This is explained by a strong modification of optical and magneto-optical properties for thin nanowires. While the coherent propagation of photons in the sample is weakly affected by thin wires due to their small volume concentration, the photon interaction with magnons is confined to the volume inside the wires where the wave polarization is strongly modified due to a huge mismatch in the dielectric constants of metallic nanowires and dielectric media. As a result, we observe that a linearly polarized beam obliquely incident upon an ultrathin Co nanowire metacrystal acquires ellipticity inside the wires that is enhanced by an order of magnitude being of the opposite sign as that compared to the case of continuous Co film or thicker Co wires. We visualize this effect by measuring the asymmetry of Stokes and anti-Stokes peaks in the Brillouin scattering spectra of light by the spin-wave modes of the wires, since the scattering can probe local electric fields [18]. We expect that our results will be instrumental in the emerging field of nonlinear spectroscopy of metamaterials [19] as well as for a design of novel structures with strong chiral responses [20] and polarization-sensitive light rooting [21-23].

\section{SAMPLE AND EXPERIMENTAL RESULTS}

An array of ultrathin Co nanowires has been grown by sequential pulsed laser deposition of $\mathrm{Co}$ and $\mathrm{CeO}_{2}$ in reductive conditions ( $P_{\text {growth }}=10^{-5}$ mbar), leading to selfassembly of Co nanowires embedded in a $\mathrm{CeO}_{2}$ matrix on a $\mathrm{SrTiO}_{3}(001)$ (SurfaceNet $\mathrm{GmbH}$ ) substrate using a quadrupled Nd:YAG laser (wavelength $266 \mathrm{~nm}$ ) operating at $10 \mathrm{~Hz}$ and a fluence in the $1-3 \mathrm{~J} \times \mathrm{cm}^{-2}$ range. More details are given in Refs. [24,25]. In order to confirm the metallic nanowire 


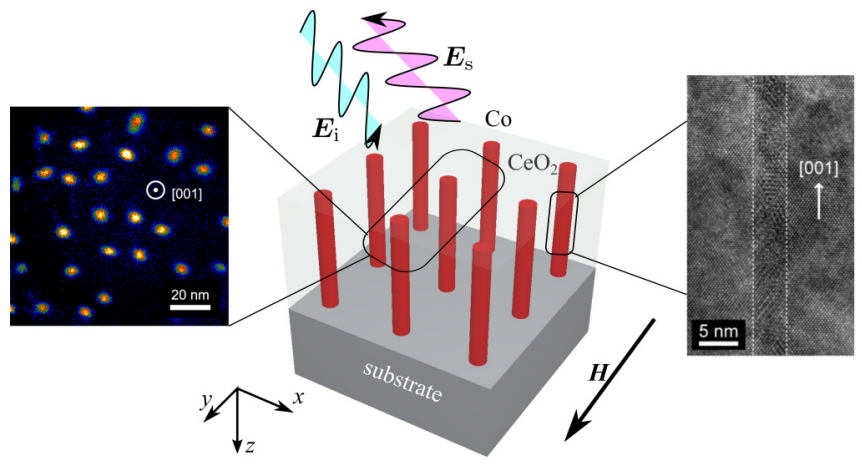

FIG. 1. (Color online) Schematics of the Co nanowires and the Brillouin scattering geometry. Incident ( $p$-polarized) and scattered ( $s$-polarized) photons and the direction of the magnetic field $\boldsymbol{H}$ are indicated. Left inset: TEM image, acquired in energy-filtered mode at the Co L edge, taken in plane-view geometry. Right inset: HRTEM image of a single nanowire taken in cross section.

formation in the sample and to determine the wire spacing and diameter, we have performed high-resolution and energyfiltered transmission electron microscopy (acquired using a JEOL JEM 2100F equipped with a field-emission gun operated at $200 \mathrm{kV}$ and a Gatan GIF spectrometer); see the right inset of Fig. 1. The wires are oriented perpendicular to the surface of the substrate and extend throughout the matrix thickness, $t$. Importantly, the technology employed is such that the wire length $h$ coincides with the film thickness $t$, i.e., $t=h$. Their length $L$ turned out to be equal to $470 \mathrm{~nm}$. The diameter $D=2 R=4.8 \pm 0.7 \mathrm{~nm}$ of the wires and the average interwire spacing $a \sim 17 \mathrm{~nm}$ were determined by collecting images in plane-view geometry (left inset in Fig. 1).

The chosen sample parameters are quite beneficial for our study. As has been shown in our earlier paper [16], the magneto-optical interaction senses the state of polarization of both interacting waves, optical and magnetic. Hence, the interpretation of the observed effects becomes much clearer when both light and magnon polarizations are well defined. This imposes specific constraints on the tailor-made sample. First, its diameter should be less than the optical skin depth, e.g., $D=5 \mathrm{~nm}$. In this case, the optical polarization inside the wire is homogeneous and thus perfectly defined, even more so if we take into consideration the fact that the near-field diffraction pattern is such that light is penetrating the nanowire isotropically from all directions. Second, such a small diameter predetermines a rather high aspect ratio, $D / L \sim 100$. A very strict selection in the frequency domain takes place then, which excludes any contributions from spin waves different from the fundamental one (referred to later as "generalized Kittel mode" [26]). The frequency and polarization state of the generalized Kittel mode can be described within a reliable and relatively simple analytical formalism. Last, but not least, to avoid cross talk, both optical and magnetic, from significantly perturbing the polarization state of the interacting magnons and photons, the sample concentration should be kept as low as possible ( $\sim 4 \%$ in the studied sample).

Now we proceed to the analysis of the spectra of Brillouin light scattering (BLS) from thermal magnons localized on ferromagnetic nanowires. The experimental arrangement is sketched in Fig. 1 and corresponds to the Damon-Eshbach geometry [27]. A magnetic field $\boldsymbol{H}$ was applied in the plane of the sample. The plane of incidence is perpendicular to the applied field. The incident laser beam has been $p$ polarized and has the wavelength $\lambda_{\text {opt }}=532 \mathrm{~nm}$. The backscattered light has been probed in $s$ polarization through a tandem Fabry-Perot interferometer (JR Sandercock product). BLS, being a particular case of nonlinear wave mixing, generates at the output two frequency-shifted optical waves, namely a downshifted one, known both in Raman and Brillouin spectroscopy as the Stokes (S) line, and an upshifted one called the anti-Stokes (AS) line. Typically, light-scattering spectra are asymmetric, i.e., the amplitudes of the $S$ and AS spectral lines are not equal. However, the physical mechanisms producing this peculiar asymmetry are completely different. In the Raman case, it is due to a greater difference between the frequencies of the $\mathrm{S}$ and AS lines which results in an appreciable asymmetry in the density of states corresponding to the frequencies $\omega+\Omega$ and $\omega-\Omega$, where $\omega$ and $\Omega$ are incoming photon and magnon frequencies, respectively. In the BLS, the frequency shifts are smaller by several orders of magnitude and entirely different physical effects are involved, namely a very particular symmetry of MO interactions. Mathematically, the symmetry of MO coupling is described by the totally antisymmetric Levi-Civita tensor $[11,13]$. As a result, the efficiency of the MO interactions is expressed via the mixed product $\boldsymbol{E}_{\mathrm{s}} \cdot\left(\boldsymbol{m} \times \boldsymbol{E}_{i}\right)$ of the polarizations of the interacting waves: the incident $\left(\boldsymbol{E}_{i}\right)$ and scattered $\left(\boldsymbol{E}_{\mathrm{s}}\right)$ optical waves and the scattering spin wave $(\boldsymbol{m})$. Importantly, in the general case of complex vector space, this mixed product is not invariant with respect to complex conjugation of the waves. In physical terms, it is the elliptical polarization that is linked to the complex vectors and the complex conjugation corresponds to the inversion of the direction of rotation of such polarization. This crucially important point will be revisited later in this work.

Experimental BLS spectra for the angles of incidence $\theta=20^{\circ}-60^{\circ}$ are presented in Figs. 2(a)-2(c). The spectra are not symmetrical: the downshifted Stokes line and the upshifted anti-Stokes line have different heights, which is not untypical of magneto-optical BLS spectra. What is really not conventional, however, is an extremely high degree of the Stokes/anti-Stokes (S/AS) asymmetry and, even more so, its inversion with respect to its "classical" pattern in which the intensity of the downshifted Stokes peak $\left|E_{\mathrm{S}}\right|^{2}$ is greater than that of the upshifted anti-Stokes one $\left|E_{\mathrm{AS}}\right|^{2}$, i.e., $\left|E_{\mathrm{S}}\right|^{2}>\left|E_{\mathrm{AS}}\right|^{2}$. The latter is illustrated in Fig. 2(d). For this comparison, we have deliberately chosen an ultrathin continuous film. The reasons are as follows. In the Damon-Eshbach geometry employed in our paper, there exists a fundamental difference between the magnonic behavior in a continuous film and in an individual nanowire. Namely, in the planar geometry, the spin wave propagates within the film and "sticks" to one of its surfaces (depending on the polarity of the saturating magnetic field). In other words, the spin wave has a nonuniform exponential profile across the film cross section ( $z$ direction in Fig. 1). This inhomogeneity produces an additional competing mechanism for S/AS scattering asymmetry. In order to single out the effect due to the mutual orientation of polarizations of the interacting waves (our case), we have chosen an ultrathin magnetic layer. More details on the scattering asymmetry in 

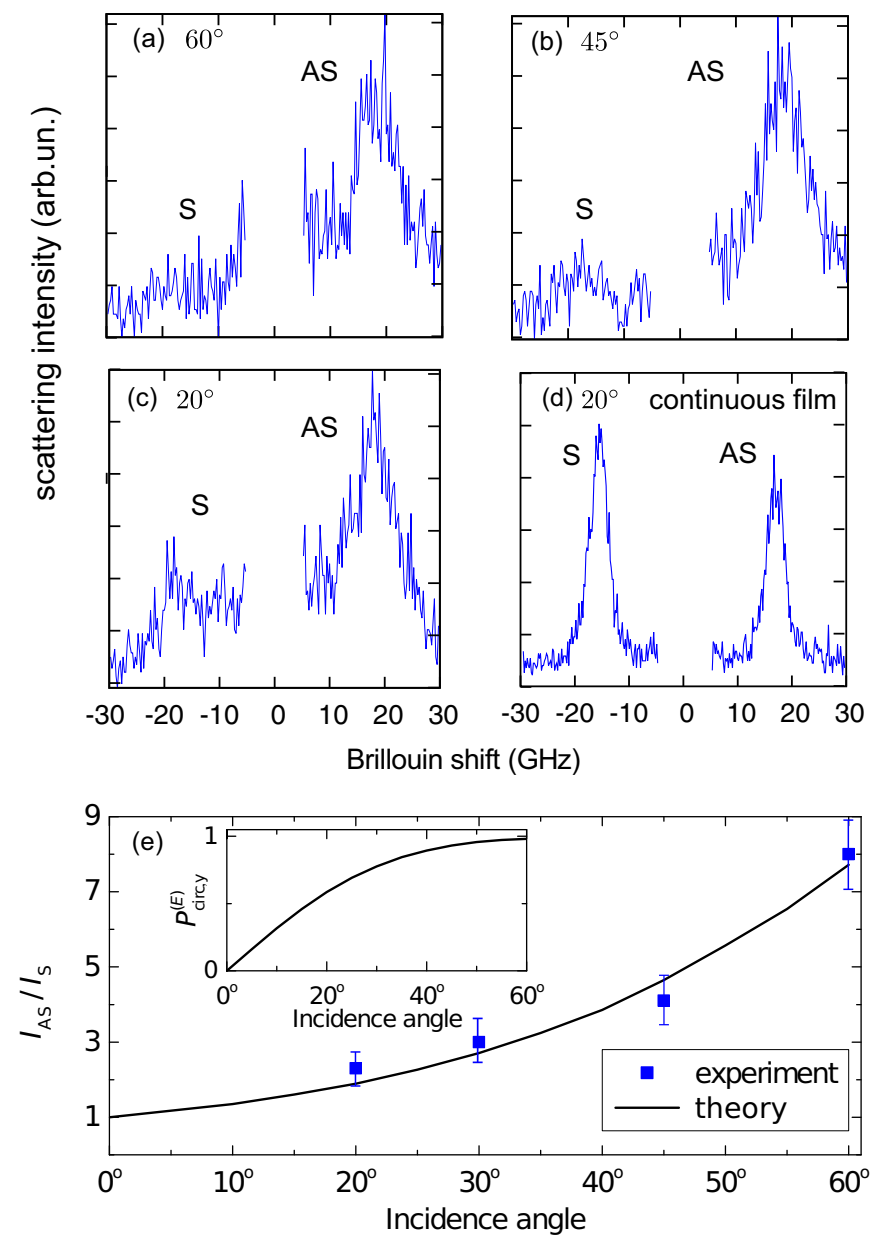

FIG. 2. (Color online) (a)-(c) Experimental Brillouin scattering spectra of the nanowire array for incidence angle $\theta=20^{\circ}, 45^{\circ}, 60^{\circ}$, with an applied in-plane magnetic field of $7 \mathrm{kOe}$. (d) Spectrum for the 1.2-nm-thick continuous Co film with the field of $6 \mathrm{kOe}$. (e) Measured (squares) and calculated (line) dependence of the ratio of Stokes and anti-Stokes spectral peak heights in the incidence angle $\theta$. The inset shows the circular polarization degree of the electric field inside the wires at $y=x=0$, averaged over the wire length. The calculation details are given in the text.

thin bulk magnetic layers can be found in the review given in Ref. [17].

This dramatic reversal of the scattering spectra asymmetry constitutes the main result of our work and will now be analyzed in detail.

\section{ANALYSIS OF THE SCATTERING ASYMMETRY}

Now we proceed to the theoretical analysis of the Stokes/anti-Stokes asymmetry in the scattering spectra. In this respect, identification of the spin-wave (SW) modes contributing to the BLS spectra is very important. The applied static magnetic field of $7 \mathrm{kOe}$ fully saturates the sample so that the magnetization in each wire is perfectly homogeneous. The latter allows a reliable theoretical description of spin-wave behavior, including explicit expressions for magnetization.

The only candidate for the role of the "effectively scattering mode" is the vertical Kittel SW modes, i.e., spin waves propagating along the wire axis with a wave number $K$ with uniform cross-section distribution (at least, in the approximation $K R \ll 1)$. The limiting case of $K=0$ corresponds to a purely magnetostatic perfectly uniform oscillation [26] and is characterized by the frequency $(\Omega / \gamma)^{2}=H\left(H-2 \pi M_{\text {eff }}\right)$. Here, $M_{\text {eff }}$ takes into account the dipolar interwire interactions between static magnetizations in individual nanowires, which makes it slightly smaller than the conventional bulk value for the cobalt, and $\gamma$ is the gyromagnetic ratio. The state of ellipticity of magnon polarization is crucial in estimating the S/AS asymmetry. Its actual value is a result of a trade-off between two trends, namely, a purely circular shape imposed by the ferromagnetic resonance and the flattening effect of dipolar interactions. The Appendix includes more details of the dispersion relation $\Omega(K)$, spatial distribution, and ellipticity of the spin waves.

For theoretical analysis of the optical properties, we use the general semiclassical theory of light scattering [31] outlined below. First, the electric field $\boldsymbol{E}_{i, \omega}(\boldsymbol{r}) e^{-i \omega t}$ of the plane wave incident upon the sample at the frequency $\omega$ is calculated. The field spatial distribution is strongly inhomogeneous and it is modified by the interaction with the wires. Second, the electromagnetic polarization induced in the wires by the interaction with the spin waves is determined [11,13]:

$$
\begin{gathered}
\boldsymbol{P}_{\mathrm{s}, \omega-\Omega}(\boldsymbol{r})=\xi \boldsymbol{m}_{\Omega}^{*}(\boldsymbol{r}) \times \boldsymbol{E}_{i, \omega}(\boldsymbol{r}) \quad(\mathrm{S}), \\
\boldsymbol{P}_{\mathrm{s}, \omega+\Omega}(\boldsymbol{r})=\xi \boldsymbol{m}_{\Omega}(\boldsymbol{r}) \times \boldsymbol{E}_{i, \omega}(\boldsymbol{r}) \quad(\mathrm{AS}) .
\end{gathered}
$$

Here, $\boldsymbol{m}_{\Omega}(\boldsymbol{r})$ is the magnetization profile of the given spin mode with the frequency $\Omega$, and $\xi$ is the interaction constant. Equations (1) and (2) correspond to Stokes and anti-Stokes scattering, respectively. Depending on whether the magnon is destructed or created, either $\boldsymbol{m}$ or $\boldsymbol{m}^{*}$ enter the expression for polarization. Clearly, the absolute values of the polarizations induced at Stokes and anti-Stokes processes can differ provided that the electromagnetic and spin waves have nonzero ellipticity. Finally, the detected field is determined as $\boldsymbol{E}_{\mathrm{s}, \omega \pm \Omega}(\boldsymbol{r})=\int d^{3} \boldsymbol{r}^{\prime} \hat{G}_{\omega \pm \Omega}\left(\boldsymbol{r}, \boldsymbol{r}^{\prime}\right) \boldsymbol{P}_{\mathrm{s}, \omega \pm \Omega}\left(\boldsymbol{r}^{\prime}\right)$, where $\hat{G}_{\omega \pm \Omega}\left(\boldsymbol{r}, \boldsymbol{r}^{\prime}\right)$ is the tensor electromagnetic Green function at the corresponding frequency.

In the experimental geometry, the incident wave is $p$ polarized, i.e., the electric field lies in the $x z$ plane (see Fig. 1). The scattered wave is detected in $s$ polarization, i.e., the electric field is parallel to the $y$ axis. We have numerically verified that $\left|G_{y y}\right| \gg\left|G_{y x}\right|$ and $\left|E_{i, y}\right| \ll\left|E_{i, x}\right|,\left|E_{i, z}\right|$ inside the wire regions, i.e., the (linear) mixing between $s$ and $p$ polarizations inside the wires is negligible. Hence, the scattering is determined by the $y$ component of the polarizations given by Eqs. (1) and (2), parallel to the detector polarization. The asymmetry between Stokes and anti-Stokes scattering can be quantified by the difference of the intensities $\left|P_{\mathrm{s}, \omega-\Omega, y}\right|^{2}-\left|P_{\mathrm{s}, \omega+\Omega, y}\right|^{2}$ that is equal to

$$
\Delta I_{\mathrm{S} / \mathrm{AS}}=|\xi|^{2}\left|\boldsymbol{m}_{\Omega}\right|^{2}\left|\boldsymbol{E}_{\text {in }}\right|^{2} P_{\text {circ }, y}^{(m)} P_{\text {circ }, y}^{(E)},
$$

where we have introduced the coordinate-dependent circular polarization degree of the wave $\boldsymbol{e}$,

$$
P_{\text {circ }, y}(\boldsymbol{r})=\frac{1}{|\boldsymbol{e}|^{2}} i\left[\boldsymbol{e}^{*} \times \boldsymbol{e}\right]_{y} \equiv \frac{2 \operatorname{Im} e_{z}^{*}(\boldsymbol{r}) e_{x}(\boldsymbol{r})}{|\boldsymbol{e}(\boldsymbol{r})|^{2}} .
$$


The quantity given by Eq. (4) changes from 1 for rightcircularly polarized fields to -1 for left-circularly polarized fields, and it vanishes for linear polarization. Equation (3) demonstrates that the Stokes-anti-Stokes asymmetry requires the nonzero ellipticity of both interacting waves. The spinwave $\boldsymbol{m}$ polarization is dominated by the ferromagnetic resonance and is fairly close to circular. Our estimations show that it is of the order of 0.8 . Another necessary ingredient for the S/AS asymmetry is the nonzero local circular polarization degree of the incident wave $P_{\text {circ }, y}^{(E)}$. Moreover, to explain the experimentally observed unexpectedly strong S/AS asymmetry, it is required that it is close to the SW ellipticity, i.e., 0.8. To justify the observed inversion of the S/AS asymmetry, the optical ellipticity should be reversed with respect to the case of a continuous Co film. Even though the incident electromagnetic wave is linearly $(p)$ polarized, the finite ellipticity is induced due to its refraction at the interfaces. This effect can be most simply illustrated for the case of a thick continuous Co film. The $p$-polarization vector inside the sample is equal to $\boldsymbol{e}_{p} \propto k_{z} \boldsymbol{e}_{x}-$ $k_{x} \boldsymbol{e}_{z}$, where $k_{x}=\omega \sin \theta / c$ is the in-plane wave vector determined by the incidence angle $\theta$ and $k_{z}=\sqrt{(\omega / c)^{2} \varepsilon_{\mathrm{Co}}-k_{x}^{2}}$. Since the permittivity of $\mathrm{Co}$ at the considered wavelength $\lambda=532 \mathrm{~nm}$ is complex, the $z$ component of the wave vector is complex as well, and the local electric field is elliptically polarized with $P_{\text {circ }} \approx-0.13$ at the incidence angle $\theta=20^{\circ}$. A crude Maxwell-Garnett model [32] for the $\mathrm{Co} / \mathrm{CeO}_{2}$ nanowire array describes it as a slightly lossy dielectric with the averaged permittivity $\bar{\varepsilon}_{\mathrm{MG}}=2\left(\varepsilon_{x x}+\varepsilon_{y y}\right) / 3+\varepsilon_{z z} / 3 \approx 5+0.4 i$. This Maxwell-Garnett approach yields $P_{\text {circ,y }}^{(E)} \approx-0.012$, i.e., even smaller and also negative circular polarization. Both of these predictions are in stark contradiction to experiment. In order to resolve this controversy, we have resorted to full-wave numerical simulation of the electric field profile inside the wires and its circular polarization degree using the CST MICROWAVE STUDIO software package. The results are presented in Fig. 3: Fig. 3(a) shows the spatial map of the circular polarization degree within the array unit cell. Figures 3(b) and 3(c) show the $z$ dependence of the polarization at the wire center and of the averaged electric field (thick black lines), respectively. The thin red lines correspond to the analytical isotropic Maxwell-Garnett model. One can see that the Maxwell-Garnett approximation describes well the distribution of the field and the polarization in the air (the region $z<0$ ), governed by the interference between incident and specularly reflected waves. The field decay in the sample $(z>0)$ along the wire axis is captured by the Maxwell-Garnett approximation as well; see Fig. 3(c). In this sense, the investigated structure behaves optically as a conventional artificial medium. However, the applicability of the Maxwell-Garnett approach does not extend any further: the circular polarization inside the wires is strongly different from the effective medium model. While the naïve Maxwell-Garnett approximation is, paradoxically enough, perfectly adequate for the wave interference and phase synchronization along the wire growth axis, it is hopelessly simplistic to predict the state of polarization within a metallic wire itself. Namely, contrary to the effective medium approximation, the circular polarization inside the wires has a positive sign and is quite large. $P_{\text {circ }}$ oscillates around the value +0.59 which greatly exceeds the values both for continuous Co film $\left(P_{\text {circ }} \approx\right.$ $-0.13)$ and for the effective medium $\left(P_{\text {circ }}=-0.012\right)$.
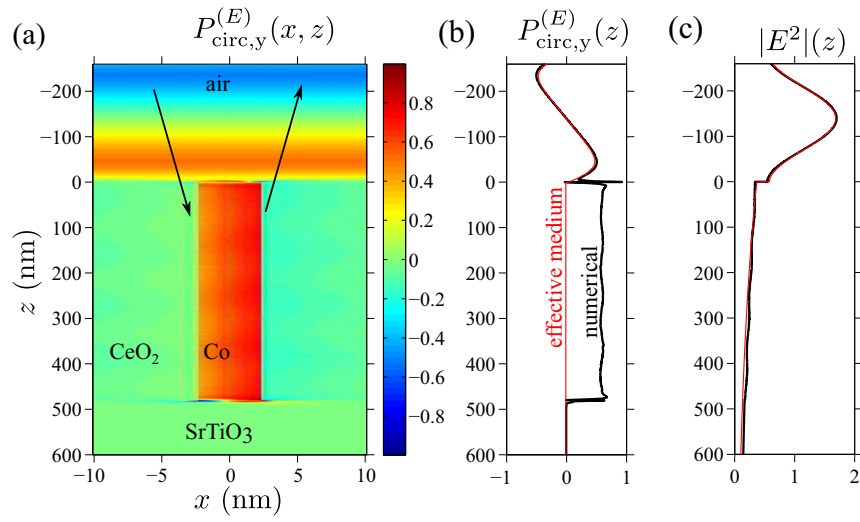

FIG. 3. (Color online) (a) Theoretically calculated spatial maps of the circular polarization of the electric field in the unit cell of Co nanowire array. The arrows show the propagation directions of incident and scattered waves. (b) The cross sections of the spatial map along the wire centers $x=y=0$, calculated numerically (black solid lines) and analytically (red thin lines). (c) Intensity of the electric field averaged over the $x$ coordinate within the unit cell at $y=0$. The calculation has been performed for $\lambda_{\text {opt }}=532 \mathrm{~nm}, \theta=20^{\circ}$, transverse magnetic (TM) polarization, $d=4.8 \mathrm{~nm}, a=20 \mathrm{~nm}$, $\varepsilon_{\mathrm{Co}}=-10.4+17.1 i$ [28], $\varepsilon_{\mathrm{CeO} 2}=4.8$ [29], and $\varepsilon_{\mathrm{SrTiO}_{3}}=6.0$ [30]. Analytical curves correspond to the isotropic Maxwell-Garnett approximation with $\varepsilon_{\mathrm{MG}}=5+0.4 i$.

These numerical findings fully explain our experimental data.

The polarization reversal can be qualitatively understood in the geometrical optics approximation by examining the refraction of a plane wave at interfaces of the structure, as shown in Figs. 4(a) and 4(b). In the case of conventional positive refraction at the surface of air and lossy metal or dielectric, one has $\operatorname{Im} k_{z}>0, k_{x}>0$, so that $\operatorname{Im} e_{p, x}>0$ and $e_{p, z}<0$ and hence Eq. (4) yields $P_{\text {circ, } y}<0$ [Fig. 4(a)]. On the other hand, the field is mostly incident upon the ultrathin wires from the side, rather than from the top face, because the top face area is much smaller than the side area [Fig. 4(b)]. As a result, the imaginary parts of the components of the field polarization vector inside the wires are swapped $\left(e_{p, x}>0\right.$ and $\left.\operatorname{Im} e_{p, z}<0\right)$ and the circular polarization sign is reversed with respect to the continuous film. The crossover between the single wire limit and the continuous film limit is illustrated by the polarization dependence on the lattice period shown in Fig. 4(c). The polarization is positive for large periods, in agreement with the geometric optics prediction (dotted line). For smaller periods, the polarization has a flat maximum at the spacings $a \sim 10 \div 15 \mathrm{~nm}$, close to the experimental ones. We attribute this maximum to the coupling between the electromagnetic modes of the wires that results in a slightly higher polarization than for an individual wire. An elaboration of the microscopic origin of the effect requires one to take into account the coupling not only between nearest wires but also the long-ranged coupling $[4,8]$. Such analysis is beyond the scope of the current study.

For even smaller periods, the polarization decreases and it changes sign as the dense wire array approaches the continuous film limit. It should be noted that although the system under investigation is seemingly simple, the number of 
(a)
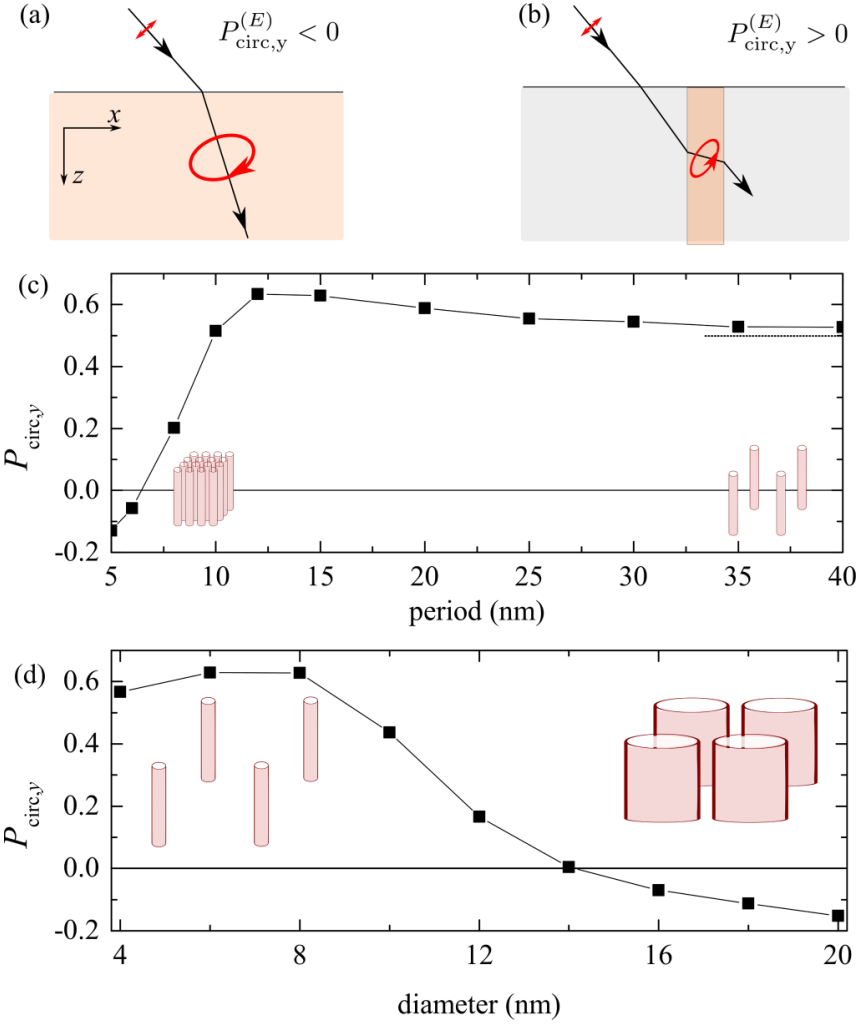

FIG. 4. (Color online) Schematic illustration of the emergence of the circular polarization of difference signs for the $p$-polarized plane wave, incident upon (a) positively refracting medium and (b) wire array. (c),(d) Dependence of averaged circular polarization inside the wires (c) on the lattice period $a$ for $D=4.7 \mathrm{~nm}$ and (d) on the wire diameter $D$ for $a=20 \mathrm{~nm}$. Dotted line in (c) shows the value of polarization for $a=400 \mathrm{~nm}$. The insets in (c) and (d) schematically illustrate the structure geometry.

parameters that actually influence its behavior is surprisingly large. Three of them are geometrical (individual wire length $L$ and diameter $D$, interwire distance in the array $a$ ). Two more parameters, namely, the optical skin depth and the characteristic magnetic length, are of purely physical nature. In other words, the problem is too complicated to be solved by consecutive consideration of innumerable options. Fortunately, the previously obtained results suggest some useful tips. Thus, the same effect can be traced by examining the calculated dependence of the polarization on the wire diameter for a fixed lattice period, shown in Fig. 4(d). In this case, the crossover corresponds to the diameter $D \approx 14 \mathrm{~nm}$ and the wire fill factor $\pi(D / 2 a)^{2} \approx 0.4$. Interestingly, approximately the same fill factor corresponds to the critical interwire period of $7 \mathrm{~nm}$, as in the previous figure. This is only possible if our system can still be regarded as an array of thin wires, i.e., the aspect ratio must be considerable $(D / L \gg 1)$ and the wire radius must not exceed the optical skin depth, which is the case in our calculations.

Good quantitative agreement between our theory and experiment is illustrated in Fig. 2(e). In this figure, we trace a ratio of the intensities of anti-Stokes and Stokes BLS lines as a function of the angle of incidence, with both experimental and theoretical values obtained using Eq. (3). Experimental errors bars were obtained by adding the relative uncertainties in the heights of the scattering peaks, estimated from the Lorentzian fits of the spectra. In the theoretical analysis, we used the values of optical ellipticity obtained from our CST MICROWAVE STUDIO numerical simulations (see Fig. 3), while the same parameter for the SW was estimated using the analytical solution for Kittel modes on an individual ferromagnetic wire. To facilitate understanding, the angular evolution of the ellipticity of the optical polarization is given in the inset of Fig. 2(e).

\section{CONCLUSIONS}

In conclusion, by employing the Brillouin light scattering tools, we have observed the effect of anomalous polarization conversion in arrays of ultrathin Co nanowires with the nanowire diameter below $5 \mathrm{~nm}$ manifested in the pronounced reversed Stokes/anti-Stokes scattering asymmetry. We have explained the latter by an unexpected circular polarization of light induced within the nanowires. In particular, the circular polarization has the opposite sign, being much larger in the absolute value than for the continuous Co film or thicker Co nanowires. This finding opens a great potential of seemingly simple nanowire arrays in manipulating light at the nanoscale. Previously, it has been shown that the wire arrays are more efficient sensors than homogeneous structures [6]. Our findings are quite general: the strong reversed circular polarization can be induced in arbitrary sparse metallic wires that are thinner than the skin depth. This indicates that the thin wires can be potentially used to probe the chiral response of the molecules put inside the arrays, which now attracts a lot of attention [20,33,34]. At the same time, our results suggest that the Brillouin spectroscopy, traditionally employed as a probe of magnon states, is an extremely sensitive technique for probing photon states as well, namely, to study polarization-resolved local electric fields in nanostructures.

\section{ACKNOWLEDGMENTS}

The authors acknowledge useful discussions with B. Jusserand and E. L. Ivchenko. We thank D. Demaille for TEM microscopy and J.-M. Guigner, IMPMC, CNRS-UPMC, for access to the TEM facilities. This work was supported by the Government of Russian Federation (Grant No. 074-U01), the Dynasty Foundation (Russia), and Australian Research Council. A.N.P. acknowledges support from RFBR and the Deutsche Forschungsgemeinschaft under the International Collaborative Research Center TRR 160. Part of this work was supported by ANR (Grant No. ANR-2011-BS04-007).

\section{APPENDIX: SPIN-MODE DISPERSION}

Here we present the details of the derivation of the results for the spin-mode frequency, which have been used in order to identify the dominantly scattering spin mode. In order to obtain the spin-mode frequency as a function of the wave vector $q$, we average the Landau-Lifshitz equation of motion together with the Maxwell magnetic flux conservation over the cross section of the cylinder, thus generalizing, for the cylindrical symmetry, the approach originally proposed for thin films by Stamps and Hillebrands [35] and revisited later in Ref. [36]. 
The applicability of this technique, as mentioned before, is limited to the case where the transversal distribution of the dynamic magnetization is close to uniform, which corresponds to the Kittel mode we are interested in. This corresponds, in the Damon-Eshbach configuration, to the lowest fundamental mode, referred to in this article as "Kittel mode" [26]. Below are sketched major steps in this calculation. A detailed account will be published elsewhere.

First, we derive the dynamic magnetic field averaged over the wire cross section. We use the coordinate system where the wire axis is directed along $z$. In the frame of the quasistatic approximation, the dynamic magnetic field $\boldsymbol{h}$ is obtained as the gradient of a potential,

$$
\boldsymbol{h}=\nabla \Phi .
$$

We assume a propagation along the nanowire axis $z$, $\Phi(x, y, z)=f(x, y) \exp (i q z)$. As the probed modes are quasiuniform across the section, we consider the dynamic magnetic field averaged over the section:

$$
\left\langle h_{x}\right\rangle=\left\langle\frac{\partial \Phi}{\partial x}\right\rangle, \quad\left\langle h_{y}\right\rangle=\left\langle\frac{\partial \Phi}{\partial y}\right\rangle, \quad\left\langle h_{z}\right\rangle=i q\langle\Phi\rangle .
$$

Next, we use the cylindrical coordinate system with $\varphi$ being the azimuthal angle in the $x y$ plane and $\rho=\sqrt{x^{2}+y^{2}}$ being the two-dimensional radius vector. Using the Stokes theorem, one can express the functions $\langle\partial \Phi / \partial x\rangle,\langle\partial \Phi / \partial y\rangle$, and $i q\langle\Phi\rangle$ via the values of $f(\rho, \varphi)$ evaluated at the nanowire surface where $\rho=R$. The function $f(R, \varphi)$ can be expanded as

$$
f(R, \varphi)=f_{0}+f_{1} \cos \varphi+f_{2} \sin \varphi .
$$

The coefficients $f_{0}, f_{1}, f_{2}$ are derived from the boundary conditions:

$$
f_{0} \approx\langle f\rangle, f_{1} \approx-2 \pi R\left\langle m_{x}\right\rangle, f_{2} \approx-2 \pi R\left\langle m_{y}\right\rangle .
$$

Thus one obtains

$$
\begin{aligned}
& \left\langle h_{x}\right\rangle=-2 \pi\left\langle m_{x}\right\rangle,\left\langle h_{y}\right\rangle=-2 \pi\left\langle m_{y}\right\rangle, \\
& \left\langle h_{z}\right\rangle=-\frac{4 \pi\left\langle m_{z}\right\rangle}{1-\frac{2}{q R} \frac{K_{0}^{\prime}(q R)}{K_{0}(q R)}},
\end{aligned}
$$

where $K_{0}$ is a modified Bessel function.

Now, the frequency from the averaged equation of motion is derived assuming an effective anisotropy energy $\pi M_{x}^{2}+$ $\pi M_{y}^{2}-K M_{z}^{2} / M^{2}$, where $K$ contains the magnetocrystalline contribution and the dipolar coupling.

First, we consider the case when the applied field is not saturating. The effective field reads $H_{y}=H-2 \pi M_{y}, H_{z}=$ $2 K M_{z} / M^{2}$. The equilibrium condition reads $H=(2 \pi+$ $\left.2 K / M^{2}\right) M_{y}$. The averaged equations of motion yields

$$
\begin{aligned}
i(\Omega / \gamma)\left\langle m_{x}\right\rangle= & M_{y}\left[\left\langle h_{z}\right\rangle-2 A q^{2}\left\langle m_{z}\right\rangle / M^{2}+2 K\left\langle m_{z}\right\rangle / M^{2}\right] \\
& -M_{z}\left[\left\langle h_{y}\right\rangle-2 A q^{2}\left\langle m_{y}\right\rangle / M^{2}\right] \\
& +\left\langle m_{y}\right\rangle H_{z}-\left\langle m_{z}\right\rangle H_{y}, \\
i(\Omega / \gamma)\left\langle m_{y}\right\rangle= & M_{z}\left[\left\langle h_{x}\right\rangle-2 A q^{2}\left\langle m_{x}\right\rangle / M^{2}\right]-\left\langle m_{x}\right\rangle H_{z}, \\
i(\Omega / \gamma)\left\langle m_{z}\right\rangle= & -M_{y}\left[\left\langle h_{x}\right\rangle-2 A q^{2}\left\langle m_{x}\right\rangle / M^{2}\right]+\left\langle m_{x}\right\rangle H_{y} .
\end{aligned}
$$

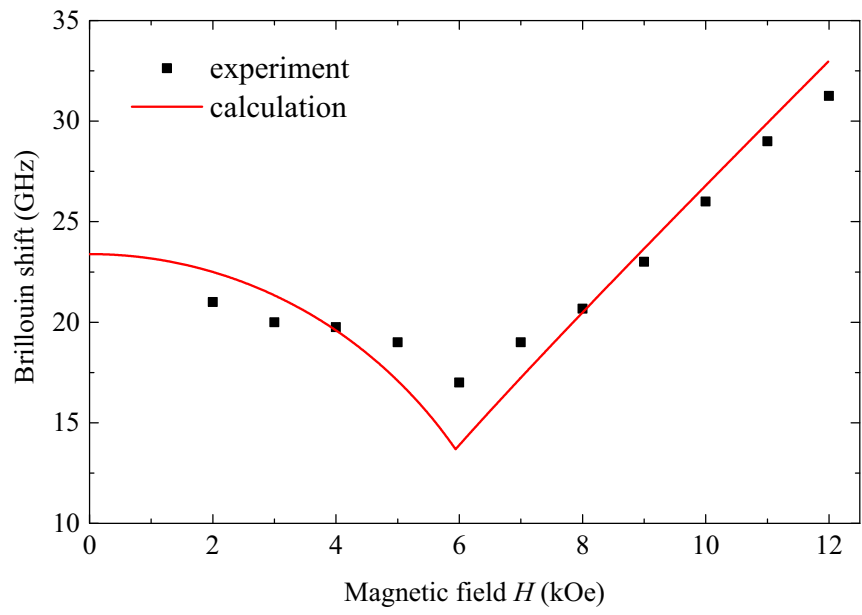

FIG. 5. (Color online) Dependence of the spin-mode frequency on the applied field strength. Squares are experimental results; the line has been calculated with the following set of parameters: $M=$ $1400 \mathrm{emu} / \mathrm{cm}^{3}, A=1.3 \times 10^{-6} \mathrm{erg} / \mathrm{cm}, \quad \gamma /(2 \pi)=3 \mathrm{GHz} / \mathrm{kOe}$, $R=2.4 \mathrm{~nm}$, and $K=-1.8 \times 10^{6} \mathrm{erg} / \mathrm{cm}^{3}$.

Replacing the averaged field components by the expressions derived in the previous part, one obtains

$$
\begin{aligned}
\left(\frac{\Omega}{\gamma}\right)^{2}= & \left\{M_{y}\left[\frac{4 \pi}{1-\frac{2}{q R} \frac{K_{0}^{\prime}(q R)}{K_{0}(q R)}}\right]+\frac{2 A q^{2}}{M^{2}}-\frac{2 K}{M^{2}}+H_{y}\right\} \\
& \times\left[M_{y}\left(2 \pi+\frac{2 q^{2} A}{M^{2}}\right)+H_{y}\right] \\
& +\left[M_{z}\left(2 \pi+\frac{2 q^{2} A}{M^{2}}\right)+H_{z}\right]^{2} .
\end{aligned}
$$

Second, we consider the case in which the applied field is saturating. Using the method presented before, one obtains

$$
\begin{aligned}
\left(\frac{\Omega}{\gamma}\right)^{2}= & \left(H+\frac{2 q^{2} A}{M}\right)\left[\frac{4 \pi}{1-\frac{2}{q R} \frac{K_{0}^{\prime}(q R)}{K_{0}(q R)}}\right. \\
& \left.+\frac{2 A q^{2}}{M}-\frac{2 K}{M}+H-2 \pi M\right] .
\end{aligned}
$$

In order to identify the origin of the dominant scattering mode, we have measured the dependence of the Brillouin shift frequency $\Omega$ versus the applied magnetic field $H$; the results are presented in Fig. 5. Interestingly, the $\Omega(H)$ curve demonstrates a pronounced minimum: the frequency decreases for low values of $H$ while displaying a clearly seen growth after passing the critical point. This characteristic softening is related to the saturation of the static magnetization. The red curve shows the calculation according to the theory above. The effective value of the wave vector $q$ of the spin wave, contributing to the Brillouin scattering, has been deduced by fitting the experimental results. According to these calculations, the wavelength of the "scattering magnon" is $2 \pi / q \sim 100 \mathrm{~nm}$.

To conclude the analysis of the spin-mode dispersion, we would like to emphasize the following. The behavior of the $\Omega(H)$ function featuring a pronounced minimum, often 
referred to as softening, is characteristic of the Kittel resonance in a Stoner-Wohlfarth particle. It has been typically observed on not too concentrated arrays of ferromagnetic nanowires since the early 2000s (see, e.g., Ref. [37]). They are quite helpful for estimating the static magnetic parameters of the system [16]. However, here we use them for the description of the dynamics of magnons traveling up and down each wire. Namely, one can obtain the exact values of the frequency $\Omega$ and wave vector $q$ of the magnon, whose contribution to the BLS is decisive.
[1] V. I. Belotelov, L. E. Kreilkamp, I. A. Akimov, A. N. Kalish, D. A. Bykov, S. Kasture, V. J. Yallapragada, A. Venu Gopal, A. M. Grishin, S. I. Khartsev, M. Nur-E-Alam, M. Vasiliev, L. L. Doskolovich, D. R. Yakovlev, K. Alameh, A. K. Zvezdin, and M. Bayer, Nat. Commun. 4, 2128 (2013).

[2] J. Y. Chin, T. Steinle, T. Wehlus, D. Dregely, T. Weiss, V. I. Belotelov, B. Stritzker, and H. Giessen, Nat. Commun. 4, 1599 (2013).

[3] M. De Luca, A. Polimeni, H. A. Fonseka, A. J. Meaney, P. C. M. Christianen, J. C. Maan, S. Paiman, H. H. Tan, F. Mura, C. Jagadish, and M. Capizzi, Nano Lett. 14, 4250 (2014).

[4] C. R. Simovski, P. A. Belov, A. V. Atrashchenko, and Y. S. Kivshar, Adv. Mater. 24, 4229 (2012).

[5] J. Yao, Z. Liu, Y. Liu, Y. Wang, C. Sun, G. Bartal, A. M. Stacy, and X. Zhang, Science 321, 930 (2008).

[6] A. V. Kabashin, P. Evans, S. Pastkovsky, W. Hendren, G. A. Wurtz, R. Atkinson, R. Pollard, V. A. Podolskiy, and A. V. Zayats, Nat. Mater. 8, 867 (2009).

[7] F. Lemoult, M. Fink, and G. Lerosey, Nat. Commun. 3, 889 (2012).

[8] P. A. Belov, R. Marqués, S. I. Maslovski, I. S. Nefedov, M. Silveirinha, C. R. Simovski, and S. A. Tretyakov, Phys. Rev. B 67, 113103 (2003).

[9] P. Ginzburg, F. J. Rodríguez-Fortuño, G. A. Wurtz, W. Dickson, A. Murphy, F. Morgan, R. J. Pollard, I. Iorsh, A. Atrashchenko, P. A. Belov, Y. S. Kivshar, A. Nevet, G. Ankonina, M. Orenstein, and A. V. Zayats, Opt. Express 21, 14907 (2013).

[10] A. Poddubny, I. Iorsh, P. Belov, and Y. Kivshar, Nat. Photon. 7, 948 (2013).

[11] M. Cardona and G. Guntherodt, Light Scattering In Solids VII: Crystal-Field And Magnetic Excitations, Topics In Applied Physics (Springer, New York, 2013).

[12] Light Scattering Spectra of Solids, edited by G. Wright (Springer-Verlag, Berlin, 1969).

[13] M. Cottam and D. Lockwood, Light Scattering in Magnetic Solids (Wiley-Interscience, New York, 1986).

[14] Z. K. Wang, H. S. Lim, H. Y. Liu, S. C. Ng, M. H. Kuok, L. L. Tay, D. J. Lockwood, M. G. Cottam, K. L. Hobbs, P. R. Larson, J. C. Keay, G. D. Lian, and M. B. Johnson, Phys. Rev. Lett. 94, 137208 (2005).

[15] Z. K. Wang, M. H. Kuok, S. C. Ng, D. J. Lockwood, M. G. Cottam, K. Nielsch, R. B. Wehrspohn, and U. Gösele, Phys. Rev. Lett. 89, 027201 (2002).

[16] A. A. Stashkevich, Y. Roussigné, P. Djemia, S. M. Chérif, P. R. Evans, A. P. Murphy, W. R. Hendren, R. Atkinson, R. J. Pollard, A. V. Zayats, G. Chaboussant, and F. Ott, Phys. Rev. B 80, 144406 (2009).
[17] P. Grünberg, in Light Scattering in Solids V, Topics in Applied Physics, Vol. 66/1, edited by M. Cardona and G. Güntherodt (Springer, Berlin Heidelberg, 1989), pp. 303-335.

[18] C. Dahl, B. Jusserand, and B. Etienne, Phys. Rev. B 51, 17211 (1995).

[19] M. R. Shcherbakov, D. N. Neshev, B. Hopkins, A. S. Shorokhov, I. Staude, E. V. Melik-Gaykazyan, M. Decker, A. A. Ezhov, A. E. Miroshnichenko, I. Brener, A. A. Fedyanin, and Y. S. Kivshar, Nano Lett. 14, 6488 (2014).

[20] Y. Tang and A. E. Cohen, Phys. Rev. Lett. 104, 163901 (2010).

[21] F. J. Rodríguez-Fortuño, G. Marino, P. Ginzburg, D. O'Connor, A. Martínez, G. A. Wurtz, and A. V. Zayats, Science 340, 328 (2013).

[22] P. V. Kapitanova, P. Ginzburg, F. J. Rodríguez-Fortuño, D. S. Filonov, P. M. Voroshilov, P. A. Belov, A. N. Poddubny, Y. S. Kivshar, G. A. Wurtz, and A. V. Zayats, Nat. Commun. 5, 3226 (2014).

[23] J. Petersen, J. Volz, and A. Rauschenbeutel, Science 346, 67 (2014).

[24] F. Vidal, Y. Zheng, P. Schio, F. J. Bonilla, M. Barturen, J. Milano, D. Demaille, E. Fonda, A. J. A. de Oliveira, and V. H. Etgens, Phys. Rev. Lett. 109, 117205 (2012).

[25] F. J. Bonilla, A. Novikova, F. Vidal, Y. Zheng, E. Fonda, D. Demaille, V. Schuler, A. Coati, A. Vlad, Y. Garreau, M. Sauvage Simkin, Y. Dumont, S. Hidki, and V. Etgens, ACS Nano 7, 4022 (2013).

[26] C. Kittel, Introduction to Solid State Physics (Wiley, New York, 1996).

[27] R. Damon and J. Eshbach, J. Phys. Chem. Solids 19, 308 (1961).

[28] P. B. Johnson and R. W. Christy, Phys. Rev. B 9, 5056 (1974).

[29] G. Hass, J. B. Ramsey, and R. Thun, J. Opt. Soc. Am. 48, 324 (1958).

[30] Handbook of Laser Science and Technology, Volume IV, Optical Material: Part 2, edited by M. J. Weber (CRC, Boca Raton, 1986).

[31] G. B. Benedek and K. Fritsch, Phys. Rev. 149, 647 (1966).

[32] A. H. Sihvola and I. V. Lindell, Prog. Electromag. Res. 6, 101 (1992).

[33] Y. Tang and A. E. Cohen, Science 332, 333 (2011).

[34] S. J. Yoo and Q.-Han Park, Phys. Rev. Lett. 114, 203003 (2015).

[35] R. L. Stamps and B. Hillebrands, Phys. Rev. B 44, 12417 (1991).

[36] F. Zighem, Y. Roussigné, S.-M. Chérif, and P. Moch, J. Phys. Condens. Matter 19, 176220 (2007).

[37] A. Encinas-Oropesa, M. Demand, L. Piraux, I. Huynen, and U. Ebels, Phys. Rev. B 63, 104415 (2001). 